\title{
A NOTE ON THE CONVERGENCE OF MOMENTS AND THE MARTINGALE CENTRAL LIMIT THEOREM
}

\author{
Esko Valkeila \\ University of Helsinki, Computing Centre, Teollisuuskatu 23 \\ SF-00510 Helsinki, Finland
}

\begin{abstract}
We study the convergence of moments of square-integrable martingales, when the martingales converge to a (mixed) normal limit.
\end{abstract}

\section{Introduction and basic facts}

1.1. Let $M^{n}, n \geq 1$, be a sequence of square-integrable local martingales defined on a filtered space $\left(\Omega^{n}, F^{n}, \mathbf{F}^{n}, \mathbf{P}^{n}\right)$, with $M_{0}^{n}=0$. Suppose that $G \subset$ $\cap_{n} F_{0}^{n}$ is a $\sigma$-algebra and $\xi$ is a random variable defined on $(\Omega, F, \mathbf{P})$ with

$$
\mathbf{E}^{G} \exp \{i t \xi\}=\exp \left\{-t^{2} \eta^{2} / 2\right\}
$$

where $\eta$ is a finite $G$-measurable random variable. Consider the following two conditions: for a fixed time $T>0$

$$
\left\langle M^{n}\right\rangle_{T} \stackrel{P}{\longrightarrow} \eta^{2}
$$

and for any $\varepsilon>0$

$$
L_{T}^{n, \varepsilon}=|x|^{2} 1_{\{|x|>\varepsilon\}} \star \nu_{T}^{n} \stackrel{P}{\longrightarrow} 0,
$$

where $\nu^{n}$ is the $\left(\mathbf{F}^{n}, \mathbf{P}^{n}\right)$-compensator of the jump measure $\mu^{n}$ of $M^{n}$. The process $L^{n, \varepsilon}$ is the Lindeberg-process.

It is well known that conditions $\left(c_{0}\right)$ and $\left(l_{0}\right)$ imply the stable convergence of $M_{T}^{n}$ to $\xi$ (see for example Liptser and Shiryaev [4; p. 316] for a more general statement proving this fact).

Instead of condition $\left(c_{0}\right)$ we assume that the following condition $\left(c_{q}\right)$ is satisfied for an integer $q>1$ :
$\left(c_{q}\right)$
$\left\langle M^{n}\right\rangle_{T} \stackrel{L^{q}(P)}{\longrightarrow} \eta^{2}$.

We will also assume that

$$
\left(\Delta M^{n}\right)_{T}^{*} \stackrel{P}{\longrightarrow} 0
$$


where $\Delta X_{t}=X_{t}-X_{t-}$ for any cadlag process $X$ and $X_{t}^{*}=\sup _{s \leq t}\left|X_{s}\right|$. Condition $(a)$ is equivalent to the following predictable condition:

$$
\left.\left.\nu^{n}(] 0, T\right] \times\{|x|>\varepsilon\}\right) \stackrel{P}{\longrightarrow} 0
$$

(see Liptser and Shiryaev [4; p. 305]). Denote by $\stackrel{S}{\longrightarrow}$ the stable convergence of random variables.

Our main result is the following

Theorem 1. Suppose that $M^{n}, n \geq 1$, is a sequence of square integrable martingales satisfying $(a)$ and $\left(c_{q}\right)$ for some $q>1$. Then the following conditions are equivalent:

a) $M_{T}^{n} \stackrel{S}{\rightarrow} \xi$ and $\mathbf{E}\left(M_{T}^{n}\right)^{2 q} \rightarrow \mathbf{E} \xi^{2 q}$,

b) $\mathbf{E}\left|\left[M^{n}\right]_{T}-\eta^{2}\right|^{q} \rightarrow 0$,

c) $\mathbf{E}|I|^{2 q} \star \nu_{T}^{n} \rightarrow 0$,

d) $\left(\left(\Delta M^{n}\right)_{T}^{*}\right)^{2 q}$ is uniformly integrable.

Theorem 1 will be proved in the third section of this paper. It was proved for discrete time martingales by Hall. He assumed condition $\left(c_{q}\right)$ and instead of $(a)$ a somewhat stronger condition, and then showed the equivalence of a), b) and c) under his conditions (see Hall and Heyde [2; pp. 70-71]).

1.2. We next introduce some notation and definitions. We assume that $(\Omega, F, \mathbf{F}, \mathbf{P})$ is a given filtered space satisfying the usual assumptions. If $X$ is a process on $(\Omega, F, \mathbf{F}, \mathbf{P})$, then $X$ is adapted to the filtration $\mathbf{F}$. Moreover, we assume that the process $X$ has right-continuous paths with left limits. If $X^{n}$ is a sequence of stochastic processes, then its properties are defined with respect to a sequence $\left(\Omega^{n}, F^{n}, \mathbf{F}^{n}, \mathbf{P}^{n}\right)$. Denote by $\mu^{X}$ the jump measure of the process $X$ and by $\nu^{X}$ the $(\mathbf{P}, \mathbf{F})$-compensator of $\mu^{X}$. If $X$ is a process, then $X^{*}$ is the increasing process $X_{t}^{*}=\sup _{s \leq t}\left|X_{s}\right|$. For terminology not explained here we refer to Jacod and Shiryaev [3].

Recall that any local martingale $M$, defined on the space $(\Omega, F, \mathbf{F}, \mathbf{P})$, admits a representation

$$
M=M^{c}+I \star\left(\mu^{M}-\nu^{M}\right),
$$

where $I_{t}(x)=x$ and $M^{c}$ is the continuous martingale part of $M$ (which depends on $\mathbf{P}$ ). Here $\star$ stands for the Lebesgue-Stieltjes integration with respect to $d x \times d t$. The quadratic variation $[M]$ of $M$ is defined by

$$
[M]_{t}=\left\langle M^{c}\right\rangle_{t}+\sum_{s \leq t}\left(\Delta X_{s}\right)^{2}
$$

If $M$ is a square-integrable local martingale, then we can define the angle bracket $\langle M\rangle$ of $M$ as the compensator of $[M]$. Recall that $\mathbf{E}\langle M\rangle_{t}=\mathbf{E}[M]_{t}=\mathbf{E} M_{t}^{2}$. 
1.3. Let $M$ be a local martingale. Recall that according to the BurkholderDavis-Gundy inequalities: for a local martingale $M$ given a stopping time $T$ and $q>2$ one has

$$
c_{q} \mathbf{E}[M]_{T}^{q / 2} \leq \mathbf{E}\left(M_{T}^{*}\right)^{q} \leq C_{q} \mathbf{E}[M]_{T}^{q / 2} .
$$

Here and in what follows $c_{q}$ and $C_{q}$ are constants with $c_{q}$ decreasing and, respectively, $C_{q}$ increasing as $q$ increases. Following versions of (1.1) were proved in $[1]$ :

$$
\begin{gathered}
c_{q} \mathbf{E}\left(\langle M\rangle_{T}^{q / 2}+\left((\Delta M)_{T}^{*}\right)^{q}\right) \leq \mathbf{E}\left(M^{*}\right)_{T}^{q} \leq C_{q} \mathbf{E}\left(\langle M\rangle_{T}^{q / 2}+\left((\Delta M)_{T}^{*}\right)^{q}\right), \\
c_{q} \mathbf{E}\left(\langle M\rangle_{T}^{q / 2}+|I|^{q} \star \nu_{T}^{M}\right) \leq \mathbf{E}\left(M_{T}^{*}\right)^{q} \leq C_{q} \mathbf{E}\left(\langle M\rangle_{T}^{q / 2}+|I|^{q} \star \nu_{T}^{M}\right) .
\end{gathered}
$$

1.4. In the next section we present some auxiliary results. A proof of Theorem 1 will be given in Section 3. In the last section we prove characterizations for the functional convergence under the additional requirement that also the moments convergence.

\section{Auxiliary results}

2.1. Suppose $M^{n} \in \mathscr{M}_{\text {loc }}^{2}$ with $M_{0}^{n}=0$. Define $\nu^{n}=\nu^{k^{n}}$ and $V^{n}=I^{2} \star \nu^{n}$. Recall that the sequence $V_{T}^{n}$ is tight if

$$
\sup _{n} \mathbf{P}\left(V_{T}^{n} \geq \lambda\right) \rightarrow 0
$$

as $\lambda \rightarrow \infty$. Define $M_{T}^{n, \varepsilon}=M_{T}^{n}+x 1_{\{|x| \leq \varepsilon\}} \star \mu_{T}^{n}$. Note that, in general, $M^{n, \varepsilon}$ is not a martingale.

Lemma 1. Suppose that the the sequence $V_{T}^{n}$ is tight for a fixed time point $T$, and that the Lindeberg condition $\left(l_{0}\right)$ holds. Then

$$
\sup _{s \leq T}\left|\left[M^{n}\right]_{s}-\left\langle M^{n}\right\rangle_{s}\right| \stackrel{P}{\longrightarrow} 0,
$$

as $n \rightarrow \infty$.

Proof. The Lindeberg condition together with the Lenglart-Rebolledo inequality imply that

$$
\left[M^{n, \varepsilon}\right]_{T}-\left[M^{n}\right]_{T} \stackrel{P}{\longrightarrow} 0 .
$$


For the predictable bracket process $\left\langle M^{n, \varepsilon}\right\rangle$ one has

$$
\left\langle M^{n, \varepsilon}\right\rangle_{T}=I^{2} 1_{\{|x| \leq \varepsilon\}} \star \nu_{T}^{n}-\sum_{s \leq T}\left(\int_{\{|x| \leq \varepsilon\}} x \nu^{n}(\{s\}, d x)\right)^{2} .
$$

Now,

$$
\int_{\{|x| \leq \varepsilon\}} x \nu^{n}(\{s\}, d x)=-\int_{\{|x|>\varepsilon\}} x \nu^{n}(\{s\}, d x)
$$

yielding

$$
\begin{aligned}
\sum_{s \leq T}\left(\int_{\{|x| \leq \varepsilon\}} x \nu^{n}(\{s\}, d x)\right)^{2} & =\sum_{s \leq T}\left(-\int_{\{|x|>\varepsilon\}} x \nu^{n}(\{s\}, d x)\right)^{2} \\
& \leq|I|^{2} 1_{\{|x|>\varepsilon\}} \star \nu_{T}^{n}
\end{aligned}
$$

Using (2.2) combined with the condition $\left(l_{0}\right)$ it is easy to see that

$$
\left\langle M^{n}\right\rangle_{T}-\left\langle M^{n, \varepsilon}\right\rangle_{T} \stackrel{P}{\longrightarrow} 0
$$

as $n \rightarrow \infty$.

To prove (2.1) we have to show that

$$
\sup _{s \leq T}\left|\left[M^{n, \varepsilon}\right]_{s}-\left\langle M^{n, \varepsilon}\right\rangle_{s}\right| \stackrel{P}{\longrightarrow} 0 .
$$

Define $Y^{n, \varepsilon}=\left[M^{n, \varepsilon}\right]-\left\langle M^{n, \varepsilon}\right\rangle$ and $\tau_{n}=\inf \left\{t \mid V_{t}^{n}>\lambda\right\}$. Clearly, the process $2|I|^{2} 1_{\{|x| \leq \varepsilon\}} \star \nu^{n}$ dominates the process $Y^{n, \varepsilon}$. We have

$$
P\left(\sup _{s \leq T}\left|Y_{s}^{n, \varepsilon}\right|>\delta\right) \leq \delta^{-2} \mathbf{E}\left(Y_{\tau_{n} \wedge t}^{n, \varepsilon}\right)^{2}+P\left(\tau_{n}<T\right)
$$

with

$$
E\left(Y_{\tau_{n} \wedge t}^{n, \varepsilon}\right)^{2} \leq 4 \varepsilon^{2}\left(\lambda+\varepsilon^{2}\right)
$$

The lemma now follows, since the right-hand side of (2.3) can be made arbitrary small by using the tighness assumption of $V_{T}^{n}$ and by an appropriate choice of $\varepsilon$.

The next lemma is obvious.

Lemma 2. Suppose that $X^{n}$ and $Y^{n}$ are sequences of random variables with

$$
X^{n} \stackrel{P}{\longrightarrow} 0 \quad \text { and } \quad \mathbf{P}\left(\left|Y^{n}\right| \geq \gamma\right) \rightarrow 0
$$

where $\gamma>0$ is a finite random variable. Then

$$
X^{n} Y^{n} \stackrel{P}{\longrightarrow} 0 .
$$


Lemma 3. Suppose

$$
\left\langle M^{n}\right\rangle_{T} \stackrel{P}{\longrightarrow} \zeta^{2}
$$

where $\zeta$ is a finite random variable and suppose the sequence

$$
\left(\left(\Delta M^{n}\right)_{T}^{*}\right)^{p}
$$

is uniformly integrable for some $p>2$. Then the following conditions are equivalent:

a) condition $\left(l_{0}\right)$ holds,

b) for any $p \geq q>2$ one has

$$
|I|^{q} \star \nu_{T}^{n} \stackrel{P}{\longrightarrow} 0
$$

c) for any $q>2$ one has

$$
|I|^{q} \star \mu_{T}^{n} \stackrel{P}{\longrightarrow} 0 .
$$

Proof. Suppose the Lindeberg condition holds. We show that condition c) holds. By (2.4) the sequence $V_{T}^{n}$ is tight. Hence by Lemma 1 we have

$$
\left[M^{n}\right]_{T} \stackrel{P}{\longrightarrow} \zeta
$$

as $n \rightarrow \infty$. Thus, for any $\varepsilon$

$$
\lim _{n} \mathbf{P}\left(|I|^{2} \star \mu_{T}^{n}>\zeta^{2}+\varepsilon\right) \rightarrow 0 .
$$

The Lindeberg condition $\left(l_{0}\right)$ implies the condition $(a)$,

$$
\left(\Delta M^{n}\right)_{T}^{*} \stackrel{P}{\longrightarrow} 0
$$

and so for any $q>2$

$$
\left(\left(\Delta M^{n}\right)_{T}^{*}\right)^{q} \stackrel{P}{\longrightarrow} 0
$$

The inequality

$$
|I|^{q} \star \mu_{T}^{n} \leq\left(\left(\Delta M^{n}\right)_{T}^{*}\right)^{q-2}|I|^{2} \star \mu_{T}^{n}
$$

together with Lemma 2 imply c).

Assume next that c) holds with $p \geq q>2$. The inequality $\left(\left(\Delta M^{n}\right)_{T}^{*}\right)^{q} \leq$ $|I|^{q} \star \mu^{n}$ can be used to show that we then have $(a)$, and hence also

$$
\left(\left(\Delta M^{n}\right)_{T}^{*}\right)^{q} \stackrel{P}{\longrightarrow} 0
$$


for any $p \geq q>2$. Since the process $|I|^{q} \star \mu^{n}$ dominates the process $|I|^{q} \star \nu^{n}$ we get, using the Lenglart-Rebolledo inequality,

$$
\mathbf{P}\left(|I|^{q} \star \nu_{T}^{n} \geq a\right) \leq \frac{1}{a} \mathbf{E}\left(|I|^{q} \star \mu_{T}^{n} \wedge\left(\left(\Delta M^{n}\right)_{T}^{*}\right)^{q}+b\right)+\mathbf{P}\left(|I|^{q} \star \mu_{T}^{n} \geq b\right) .
$$

The last inequality combined with (2.5) and (a) gives b).

Assume finally that $b$ ) holds. Using the inequality

$$
\varepsilon^{q-2} \int_{0}^{T} \int_{\{|x|>\varepsilon\}}|x|^{2} \nu^{n}(d s, d x) \leq|I|^{q} \star \nu_{T}^{n}
$$

we see that b) implies a).

Remark 1. Note that in the previous lemma we have proved the following three implications:

a) 2.4) \& $\left.\left(l_{0}\right) \Rightarrow c\right)$

b) c) \& (2.5) $\Rightarrow$ b)

c) b) $\Rightarrow\left(l_{0}\right)$.

Lemma 4. Let $M^{n}$ be a sequence of square-integrable local martingales and let $T$ be a stopping time. The following conditions are equivalent:

a) the sequence $\left|M_{T}^{n}\right|^{p}$ is uniformly integrable for $p>2$,

b) the sequence $\left(\left(M^{n}\right)_{T}^{*}\right)^{p}$ is uniformly integrable for $p>2$.

Proof. Assume b). Since $\left|M_{T}^{n}\right| \leq\left(M^{n}\right)_{T}^{*}$ it is clear that a) holds.

Assume a). We first note that the sequence $\mathbf{E}\left(\left(M^{n}\right)_{T}^{*}\right)^{p}$ is bounded, since

$$
\mathbf{E}\left(\left(M^{n}\right)_{T}^{*}\right)^{p} \leq C_{p}\left|M_{T}^{n}\right|^{p}
$$

For $c>0$ put $A_{c}^{n}=\left\{\left(M^{*}\right)_{T}^{n} \geq c\right\}$. Recall that from Doob's inequality one gets

$$
\mathbf{P}\left(A_{c}^{n}\right) \leq \frac{1}{c} \mathbf{E} 1_{A_{c}^{n}}\left|M_{T}^{n}\right|
$$

yielding

$$
\begin{aligned}
\mathbf{E}\left(\left(\left(M^{*}\right)_{T}^{n}\right)^{p} 1_{A_{c^{1 / p}}^{n}}\right) & =\int_{c^{1 / p}}^{\infty} \mathbf{P}\left(A_{a^{1 / p}}^{n}\right) d a \leq \mathbf{E}\left|M_{T}^{n}\right| \int_{c}^{\left(\left(M^{*}\right)_{T}^{n}\right)^{p}} a^{-1 / p} d a \\
& =\frac{p}{p-1} \mathbf{E}\left|M_{T}^{n}\right|\left(\left(M^{*}\right)_{T}^{n}\right)^{p} 1_{A_{c^{1 / p}}^{n}} .
\end{aligned}
$$

Hölder's inequality then gives

$$
\mathbf{E}\left(\left(\left(M^{*}\right)_{T}^{n}\right)^{p} 1_{A_{c^{1 / p}}^{n}}\right) \leq C_{p} \mathbf{E}\left(\left|M^{n}\right|_{T}^{p} 1_{A_{c}^{n}}\right) .
$$

Since $\sup _{n} \lim _{c \rightarrow \infty} P\left(A_{c}^{n}\right) \rightarrow 0$ and since the sequence $\left|M_{T}^{n}\right|$ is uniformly integrable, we have

$$
\sup _{n} \lim _{c \rightarrow \infty} \mathbf{E}\left(\left|M^{n}\right|_{T}^{p} 1_{A_{c}^{n}}\right)=0 .
$$

It clearly follows from (2.6) that the same is true for $\left(\left(M_{T}^{n}\right)^{*}\right)^{p}$, yielding b).

In what follows we often make use of the following principle. 
Lemma 5. Suppose that $X^{n}$ is a sequence of random variables with

$$
X^{n} \stackrel{d}{\longrightarrow} \gamma
$$

where $\gamma$ is a random variable having all the moments and $\stackrel{d}{\longrightarrow}$ stands for convergence in distribution; and suppose

$$
\mathbf{E}\left(X^{n}\right)^{2} \rightarrow \mathbf{E} \gamma^{2} .
$$

If for $q>2$ the sequence $\mathbf{E}\left(X^{n}\right)^{q}$ is bounded, then we have

$$
\mathbf{E}\left(X^{n}\right)^{q} \rightarrow \mathbf{E} \gamma^{q} .
$$

Proof. Introduce the following two topologies $\tau$ and $\sigma$ :

a sequence $Y^{n}$ converges with respect to $\tau$, if $Y^{n}$ converges in distribution to a random variable $\kappa$ and $\mathbf{E}\left(Y^{n}\right)^{2} \rightarrow \mathbf{E} \kappa^{2}$,

a sequence $Y^{n}$ converges with respect to $\sigma$, if $Y^{n}$ converges in distribution to a random variable $\kappa$ and $\mathbf{E}\left(Y^{n}\right)^{q} \rightarrow \mathbf{E} \kappa^{q}$

It is clear that convergence with respect to $\sigma$ is stronger than convergence with respect to $\tau$.

After these definitions we continue with the proof. If the sequence $\mathbf{E}\left(X^{n}\right)^{q}$ is bounded, there is a subsequence with $\mathbf{E}\left(X^{n_{k}}\right)^{q} \rightarrow c$. Then, obviously $c=\mathbf{E} \gamma^{q}$. This also shows that there are no other possible cluster points for the sequence $\mathbf{E}\left(X^{n}\right)^{q}$, finishing the proof.

Remark 2. The above lemma is a modification of Zolotarev [5; pp. 100-101].

\section{Main results and proofs}

3.1. We start the proof of Theorem 1 with

Theorem 2. Suppose $M^{n}$ is a sequence of square-integrable local martingales for which conditions $\left(c_{1}\right)$ and $(a)$ hold. Then the following conditions are equivalent:

a) $\mathbf{E}\left|\left[M^{n}\right]_{T}-\eta^{2}\right| \rightarrow 0$,

b) Lindeberg's condition $\left(l_{0}\right)$ holds in $L^{1}(P)$,

c) $\left(\left(\Delta M^{n}\right)_{T}^{*}\right)^{2}$ is uniformly integrable,

d) $M_{T}^{n} \stackrel{S}{\longrightarrow} \xi$ and $\mathbf{E} M_{T}^{2} \rightarrow \mathbf{E} \xi^{2}$.

Proof. Assume a). Then

$$
\left[M^{n}\right]_{T}-\left[M^{n, \varepsilon}\right]=|I|^{2} 1_{\{|x| \geq \varepsilon\}} \star \mu_{T}^{n} .
$$


Moreover,

$$
|I|^{2} 1_{\{|x| \geq \varepsilon\}} \star \mu_{T}^{n} \leq\left(\Delta M^{n}\right)_{T}^{*} \frac{1}{\varepsilon}|I|^{2} 1_{\{|x| \geq \varepsilon\}} \star \mu_{T}^{n} .
$$

Thus, by a) and condition $(a)$ we get $|I|^{2} 1_{\{|x| \gamma e \varepsilon\}} \star \mu_{T}^{n} \stackrel{P}{\longrightarrow} 0$. By a) the sequence $\left[M^{n}\right]_{T}$ is uniformly integrable. Thus, the same is true also for the sequence $|I|^{2} 1_{\{|x| \geq \varepsilon\}} \star \mu_{T}^{n}$. Hence $\mathbf{E}|I|^{2} 1_{\{|x| \geq \varepsilon\}} \star \nu_{T}^{n} \rightarrow 0$, proving b).

Assume b). Since $|I|^{2} 1_{\{|x|>\varepsilon\}} \star \mu_{T}^{n} \stackrel{P}{\longrightarrow} 0$, the sequence $|I|^{2} 1_{\{|x|>\varepsilon\}} \star \mu_{T}^{n}$ is uniformly integrable. Moreover, since $\left(\Delta M^{n}\right)^{2} \leq \varepsilon^{2}+|I|^{2} 1_{\{|x|>\varepsilon\}} \star \mu_{T}^{n}$ also the sequence $\left(\left(\Delta M_{T}^{n}\right)^{*}\right)^{2}$ is uniformly integrable, giving c).

Assume c). We have

$$
|I|^{2} 1_{\{|x|>\varepsilon\}} \star \mu_{T}^{n} \leq\left(\left(M^{n}\right)_{T}^{*}\right) \frac{1}{\varepsilon}|I|^{2} 1_{\{|x|>\varepsilon\}} \star \mu_{T}^{n} .
$$

Note that the random variable $|I|^{2} 1_{\{|x|>\varepsilon\}} \star \mu_{T}^{n}$ is integrable by $\left(c_{1}\right)$ and by the equality $\mathbf{E}\left[M^{n}\right]_{T}=\mathbf{E}\left\langle M^{n}\right\rangle_{T}$. This together with $(a)$ then implies that

$$
|I|^{2} 1_{\{|x|>\varepsilon\}} \star \mu_{T}^{n} \stackrel{P}{\longrightarrow} 0 .
$$

By the Lenglart-Rebolledo inequality we get

$$
\begin{aligned}
\mathbf{P}\left(|I|^{2} 1_{\{|x|>\varepsilon\}} \star \nu_{T}^{n}>a\right) \leq & \frac{1}{a} \mathbf{E}\left(b+\left(\left(\Delta M^{n}\right)_{T}^{*}\right)^{2}\right) \wedge|I|^{2} 1_{\{|x|>\varepsilon\}} \star \mu_{T}^{n} \\
& +\mathbf{P}\left(|I|^{2} 1_{\{|x|>\varepsilon\}} \star \mu_{T}^{n} \geq b\right) .
\end{aligned}
$$

This, together with $(2.7),(a)$ and c) shows that the Lindeberg condition $\left(l_{0}\right)$ is satisfied. So by Lemma 1

$$
\left[M^{n}\right]_{T} \stackrel{P}{\longrightarrow} \eta^{2} .
$$

But by (2.7) $\lim \mathbf{E}\left[M^{n}\right]_{T}=\mathbf{E} \xi^{2}$ and so the sequnece $\left[M^{n}\right]_{T}$ is uniformly integrable, giving a).

Assume b). We have then $\left(l_{0}\right)$ and $\left(c_{0}\right)$ implying $M_{T}^{n} \stackrel{S}{\longrightarrow} \xi$. By $\left(c_{1}\right)$ we have $\mathbf{E}\left(M^{n}\right)_{T}^{2} \rightarrow \mathbf{E} \xi^{2}$, giving d).

Assume d). Then the sequence $\left(M_{T}^{n}\right)^{2}$ is uniformly integrable. By Lemma 4 the same holds for the sequence $\left(\left(M^{n}\right)_{T}^{*}\right)^{2}$. Thus, also the sequence $\left(\left(\Delta M^{n}\right)_{T}^{*}\right)^{2}$ is uniformly integrable, giving $c)$.

3.2. We prove now Theorem 1 . Thus, we assume that for some $q>1$ the conditions $\left(c_{q}\right)$ and $(a)$ hold. Note that $\left(c_{q}\right)$ implies $\left(c_{1}\right)$ and hence Theorem 2 is applicable. 
Assume a). By Theorem 2 we have $\mathbf{E}\left|\left[M^{n}\right]_{T}-\eta^{2}\right| \rightarrow 0$. The sequence $\mathbf{E}\left(\left(M^{n}\right)_{T}^{*}\right)^{2 q}$ is by a) bounded, and b) follows then by using similar arguments as in the the proof of Lemma 5.

Assume b). Then the sequence $\left[M^{n}\right]_{T}^{q}$ is uniformly integrable. The inequality $\left(\left(\Delta M^{n}\right)_{T}^{*}\right)^{2 q} \leq\left[M^{n}\right]_{T}^{q}$ then gives d).

Assume d). By Theorem $2\left(l_{0}\right)$ holds, and hence by Lemma 3

$$
|I|^{2 q} \star \nu_{T}^{n} \stackrel{P}{\longrightarrow} 0 .
$$

From d), (1.2), (1.3) and $\left(c_{q}\right)$ we get that the sequence $|I|^{2 q} \star \nu_{T}^{n}$ is bounded in $L^{1}(P)$. Using similar arguments as in the proof of Lemma 5 we then see that c) holds.

Assume c). We then have $\mathbf{E} L_{T}^{n, \varepsilon} \rightarrow 0$ (cf. Remark 2.1. c)), and by Theorem 2 we get $M_{T}^{n} \stackrel{S}{\longrightarrow} \xi$ and $\mathbf{E}\left(M_{T}^{n}\right)^{2} \rightarrow \mathbf{E} \xi^{2}$. Now use (1.3) together with c) and $\left(c_{q}\right)$ to check that $\mathbf{E}\left(M_{T}^{n}\right)^{2 q}$ is bounded. Hence a) follows from Lemma 2.5, finishing the proof of Theorem 1 .

For the discrete time we have the following

Corollary 1. Suppose $M_{i}^{n}, i=1, \ldots, n_{k}$, is a sequence of square integrable martingales with

$$
\left[M^{n}\right]_{n_{k}}=\sum_{i=1}^{n_{k}}\left(\Delta M_{i}^{n}\right)^{2}
$$

and

$$
\left\langle M^{n}\right\rangle_{n_{k}}=\sum_{i=1}^{n_{k}} E^{F_{i-1}^{n}}\left(\Delta M_{i}^{n}\right)^{2},
$$

where $\Delta M_{i}^{n}=M_{i}^{n}-M_{i-1}^{n}$. Assume that $\left(c_{1}\right)$ and $(a)$ hold. Then Theorem 2 is true. If $\left(c_{q}\right)$ and $(a)$ hold, Theorem 1 is true. Condition ( $a$ ) can be replaced by the corresponding condition $\left(a^{*}\right)$ :

$$
\sum_{i=1}^{n_{k}} \mathbf{P}\left(\left|\Delta M_{i}^{n}\right|>\varepsilon \mid F_{i-1}^{n}\right) \stackrel{P}{\longrightarrow} 0
$$

Remark 3. Hall (see Hall and Heyde [2; pp. 70-71]) assumes instead of (a) the following condition:

$$
\max _{i} \mathbf{E}^{F_{i-1}^{n}}\left(\Delta M_{i}^{n}\right)^{2} \stackrel{P}{\longrightarrow} 0 .
$$

It is easy to see that (3.1) implies (a). Hall also assumes that $\mathbf{E}\left(M_{n_{k}}^{n}\right)^{2}=1$. 


\section{On the functional central limit theorem}

4.1. Denote by $\stackrel{\mathscr{L}}{\longrightarrow}$ the weak convergence in the Skorohod space. In this section we study the moment convergence under the functional central limit theorem. Assume that $D$ is a dense subset of $R_{+}$. It is well known that if we have for each $T \in D\left(c_{0}(D)\right)$ :

$$
\left\langle M^{n}\right\rangle_{T} \stackrel{P}{\longrightarrow} C_{T}
$$

and for each $T \in D\left(l_{0}(D)\right)$ :

$$
L_{T}^{n, \varepsilon} \stackrel{P}{\longrightarrow} 0
$$

then

$$
M^{n} \stackrel{\mathscr{L}}{\longrightarrow} M
$$

where $M$ is a continuous Gaussian martingale with $\langle M\rangle_{T}=C_{T}$ and $C$ is an increasing continuous function. Instead of (4.1) consider the following condition, valid for each $T \in D$ and some $q \geq 1$ :

$$
M^{n} \stackrel{\mathscr{L}}{\longrightarrow} M \quad \text { and } \quad \mathbf{E}\left(M_{T}^{n}\right)^{2 q} \rightarrow \mathbf{E} M_{T}^{2 q} .
$$

We have the following:

Theorem 3. The following conditions are equivalent:

a) $\left(\mathscr{L}_{q}\right)$ holds for some $q \geq 1$;

b) for $T \in D$ one has $\mathbf{E}\left|\left[M^{n}\right]_{T}-C_{T}\right|^{q} \rightarrow 0$;

c) for $T \in D$ one has $\mathbf{E}\left|\left\langle M^{n}\right\rangle_{T}-C_{T}\right|^{q} \rightarrow 0$ and $\mathbf{E}\left(\left(\Delta M^{n}\right)_{T}^{*}\right)^{2 q} \rightarrow 0$.

If $q>1$ then $\mathrm{c}$ ) is equivalent to

d) for $T \in D$ one has $\mathbf{E}\left|\left\langle M^{n}\right\rangle_{T}-C_{T}\right|^{q} \rightarrow 0$ and $\mathbf{E}|I|^{2 q} \star \nu_{T}^{n} \rightarrow 0$.

Proof. Assume a) and $q=1$. Then

$$
\left|\left[M^{n}\right]_{T}-C_{T}\right| \stackrel{P}{\longrightarrow} 0
$$

(see Liptser and Shiryaev [4; p. 417]). Condition b) then follows from $E\left(M_{T}^{n}\right)^{2} \rightarrow$ $C_{T}$. If $q>1$ we still have (4.2). The fact that (4.2) is valid also in $L^{q}(P)$ follows from Theorem 1 , giving $b$ ).

Assume b). Then, the continuity of $C$ implies that

$$
\left(\Delta M^{n}\right)_{T}^{*} \stackrel{P}{\longrightarrow} 0 .
$$


Because $\left(\left(\Delta M^{n}\right)_{T}^{*}\right)^{2} \leq\left[M^{n}\right]_{T}$ and $\left[M^{n}\right]_{T}^{q}$ is uniformly integrable by b), we get that (4.3) holds in $L^{2 q}(P)$. Since for any $\delta>0$

$$
x^{2} 1_{\{|x|>\delta\}} \star \mu_{T}^{n} \leq \frac{\left(\Delta M^{n}\right)_{T}^{*}}{\delta}\left[M^{n}\right]_{T},
$$

we get by b), (4.3) and Lemma 2 that $x^{2} 1_{\{|x|>\delta\}} \star \mu_{T}^{n} \stackrel{P}{\longrightarrow} 0$. The LenglartRebolledo inequality and the fact that (4.3) holds in $L^{2}(P)$ then imply that the Lindeberg condition $\left(l_{0}\right)(D)$ holds. So, by Lemma 1 , we have

$$
\left|\left\langle M^{n}\right\rangle_{T}-C_{T}\right| \stackrel{P}{\longrightarrow} 0 .
$$

Using b) it is easy to check that (4.4) holds in $L(P)$ and as in the proof of Theorem 1 we get that (4.4) holds in $L^{q}(P)$, giving $\left.\mathrm{c}\right)$.

Assume c). By Theorem 2 we have $\left(l_{0}\right)(D)$, and hence $M^{n} \stackrel{\mathscr{L}}{\longrightarrow} M$. The rest of a) follows then from Theorem 2 for $q=1$ and from Theorem 1 for $q=2$.

Assume d) and $q>1$. It is obvious that also c) holds.

Assume c) and $q>1$. Then d) follows as in the proof of Theorem 1.

Remark 4. Condition $\mathscr{L}_{q}$ is equivalent to

$$
M^{n} \stackrel{\mathscr{L}}{\longrightarrow} M \quad \text { and } \quad\left(M_{T}^{n}\right)^{2 q} \text { is uniformly integrable. }
$$

\section{References}

[1] Dzhaparidze, K., and E. Valkeila: On the Hellinger-type distances for filtered experiments. - Probab. Theory Related Fields 85, 1990, 105-117.

[2] Hall, P., and C. Heyde: Martingale limit theory. - Academic Press 1980, New York.

[3] JACOD, J., and A.N. Shiryaev: Limit theorems for stochastic processes. - SpringerVerlag, Berlin-Heidelberg-New York, 1987.

[4] Liptser, R.S., and A.N. Shiryaev: Theory of martingales. - Nauka, Moscow, 1986 (Russian).

[5] Zolotarev, V.M.: Modern theory of summing independent random variables. - Nauka, Moscow, 1986 (Russian). 